\title{
Research Article \\ Existence Results for a Fractional Equation with State-Dependent Delay
}

\author{
José Paulo Carvalho dos Santos, ${ }^{1}$ Claudio Cuevas, ${ }^{2}$ \\ and Bruno de Andrade ${ }^{3}$ \\ ${ }^{1}$ Instituto de Ciências Exatas, Universidade Federal de Alfenas, 37130-000 Alfenas, MG, Brazil \\ ${ }^{2}$ Departamento de Matemática, Universidade Federal de Pernambuco, 50540-740 Recife, PE, Brazil \\ ${ }^{3}$ Departamento de Matemática, ICMC, USP-São Carlos, 13569-970 São Carlos, SP, Brazil
}

Correspondence should be addressed to Bruno de Andrade, bruno00luis@gmail.com

Received 26 August 2010; Revised 14 January 2011; Accepted 7 March 2011

Academic Editor: Dumitru Baleanu

Copyright (C) 2011 José Paulo Carvalho dos Santos et al. This is an open access article distributed under the Creative Commons Attribution License, which permits unrestricted use, distribution, and reproduction in any medium, provided the original work is properly cited.

We provide sufficient conditions for the existence of mild solutions for a class of abstract fractional integrodifferential equations with state-dependent delay. A concrete application in the theory of heat conduction in materials with memory is also given.

\section{Introduction}

In the last two decades, the theory of fractional calculus has gained importance and popularity, due to its wide range of applications in varied fields of sciences and engineering as viscoelasticity, electrochemistry of corrosion, chemical physics, optics and signal processing, and so on. The main object of this paper is to provide sufficient conditions for the existence of mild solutions for a class of abstract partial neutral integrodifferential equations with statedependent delay described in the form

$$
\begin{gathered}
D_{t}^{\alpha} x(t)=A x(t)+\int_{0}^{t} B(t-s) x(s) d s+f\left(t, x_{\rho\left(t, x_{t}\right)}\right), \quad \alpha \in(1,2), \\
x_{0}=\varphi \in ß, \quad x^{\prime}(0)=0,
\end{gathered}
$$

where $t \in I=[0, b], A,(B(t))_{t \geq 0}$ are closed linear operators defined on a common domain which is dense in a Banach space $(X,\|\cdot\|)$, and $D_{t}^{\alpha} h(t)$ represent the Caputo derivative of 
$\alpha>0$ defined by

$$
D_{t}^{\alpha} h(t):=\int_{0}^{t} g_{n-\alpha}(t-s) \frac{d^{n}}{d s^{n}} h(s) d s
$$

where $n$ is the smallest integer greater than or equal to $\alpha$ and $g_{\beta}(t):=t^{\beta-1} / \Gamma(\beta), t>0, \beta \geq 0$. The history $x_{t}:(-\infty, 0] \rightarrow X$ given by $x_{t}(\theta)=x(t+\theta)$ belongs to some abstract phase space $\mathbb{B}$ defined axiomatically, and $f:[0, b] \times \mathcal{B} \rightarrow X$ and $\rho:[0, b] \times \mathcal{B} \rightarrow(-\infty, b]$ are appropriated functions.

Functional differential equations with state-dependent delay appear frequently in applications as model of equations, and for this reason, the study of this type of equations has received great attention in the last years. The literature devoted to this subject is concerned fundamentally with first-order functional differential equations for which the state belong to some finite dimensional space, see among other works, [1-10]. The problem of the existence of solutions for partial functional differential equations with state-dependent delay has been recently treated in the literature in [11-15]. On the other hand, existence and uniqueness of solutions for fractional differential equations with delay was recently studied by Maraaba et al. in [16, 17]. In [18], the authors provide sufficient conditions for the existence of mild solutions for a class of fractional integrodifferential equations with state-dependent delay. However, the existence of mild solutions for the class of fractional integrodifferential equations with state-dependent delay of the form (1.1)-(1.2) seems to be an unread topic.

The plan of this paper is as follows. The second section provides the necessary definitions and preliminary results. In particular, we review some of the standard properties of the $\alpha$-resolvent operators (see Theorem 2.11). We also employ an axiomatic definition for the phase space $B$ which is similar to those introduced in [19]. In the third section, we use fixed-point theory to establish the existence of mild solutions for the problem (1.1). To show how easily our existence theory can be used in practice, in the fourth section, we illustrate an example.

\section{Preliminaries}

In what follows, we recall some definitions, notations, and results that we need in the sequel. Throughout this paper, $(X,\|\cdot\|)$ is a Banach space, and $A, B(t), t \geq 0$, are closed linear operators defined on a common domain $\mathscr{\Phi}=D(A)$ which is dense in $X$; the notations $\rho(A)$ and $[D(A)]$ represent the resolvent set of the operator $A$ and the domain of $A$ endowed with the graph norm, respectively. For $\lambda \in \rho(A)$, we fix $R(\lambda, A):=(\lambda-A)^{-1}$, and we represent by $\|R(\lambda, A)\|$ the norm of $R(\lambda, A)$ in $\mathcal{L}(X)$. Let $\left(Z,\|\cdot\|_{Z}\right)$ and $\left(W,\|\cdot\|_{W}\right)$ be Banach spaces. In this paper, the notation $\mathcal{L}(Z, W)$ stands for the Banach space of bounded linear operators from $Z$ into $W$ endowed with the uniform operator topology, and we abbreviate this notation to $\mathcal{L}(Z)$ when $Z=W$. Furthermore, for appropriate functions $K:[0, \infty) \rightarrow Z$, the notation $\widehat{K}$ denotes the Laplace transform of $K$. The notation $B_{r}(x, Z)$ stands for the closed ball with center at $x$ and radius $r>0$ in $Z$. On the other hand, for a bounded function $\gamma:[0, a] \rightarrow Z$ and $t \in[0, a]$, the notation $\|\gamma\|_{Z, t}$ is given by

$$
\|\gamma\|_{Z, t}=\sup \left\{\|\gamma(s)\|_{Z}: s \in[0, t]\right\},
$$

and we simplify this notation to $\|\gamma\|_{t}$ when no confusion about the space $Z$ arises. 
We will define the phase space $B$ axiomatically, using ideas and notations developed in [19]. More precisely, $\mathbb{B}$ will denote the vector space of functions defined from $(-\infty, 0]$ into $X$ endowed with a seminorm denoted $\|\cdot\|_{\mathcal{B}}$, and such that the following axioms hold.

(A) If $x:(-\infty, \sigma+b) \rightarrow X, b>0, \sigma \in \mathbb{R}$ is continuous on $[\sigma, \sigma+b)$ and $x_{\sigma} \in \mathbb{B}$, then for every $t \in[\sigma, \sigma+b)$ the following conditions hold:

(i) $x_{t}$ is in $\mathbb{B}$,

(ii) $\|x(t)\| \leq H\left\|x_{t}\right\|_{B}$,

(iii) $\left\|x_{t}\right\|_{\mathcal{B}} \leq K(t-\sigma) \sup \{\|x(s)\|: \sigma \leq s \leq t\}+M(t-\sigma)\left\|x_{\sigma}\right\|_{B_{B}}$

where $H>0$ is a constant; $K, M:[0, \infty) \rightarrow[1, \infty), K(\cdot)$ is continuous, $M(\cdot)$ is locally bounded, and $H, K, M$ are independent of $x(\cdot)$.

(A1) For the function $x(\cdot)$ in (A), the function $t \rightarrow x_{t}$ is continuous from $[\sigma, \sigma+b)$ into B.

(B) The space $B$ is complete.

Example 2.1 (the phase space $\left.C_{r} \times L^{p}(g, X)\right)$. Let $r \geq 0,1 \leq p<\infty$, and let $g:(-\infty,-r] \rightarrow \mathbb{R}$ be a nonnegative measurable function which satisfies the conditions (g-5), (g-6) in the terminology of [19]. Briefly, this means that $g$ is locally integrable and there exists a nonnegative, locally bounded function $\gamma$ on $(-\infty, 0]$, such that $g(\xi+\theta) \leq \gamma(\xi) g(\theta)$, for all $\xi \leq 0$ and $\theta \in(-\infty,-r) \backslash N_{\xi}$, where $N_{\xi} \subseteq(-\infty,-r)$ is a set with Lebesgue measure zero. The space $C_{r} \times L^{p}(g, X)$ consists of all classes of functions $\varphi:(-\infty, 0] \rightarrow X$, such that $\varphi$ is continuous on $[-r, 0]$, Lebesgue-measurable, and $g\|\varphi\|^{p}$ is Lebesgue integrable on $(-\infty,-r)$. The seminorm in $C_{r} \times L^{p}(g, X)$ is defined by

$$
\|\varphi\|_{\mathcal{B}}:=\sup \{\|\varphi(\theta)\|:-r \leq \theta \leq 0\}+\left(\int_{-\infty}^{-r} g(\theta)\|\varphi(\theta)\|^{p} d \theta\right)^{1 / p}
$$

The space $B=C_{r} \times L^{p}(g ; X)$ satisfies axioms (A), (A1), (B). Moreover, when $r=0$ and $p=2$, we can take $H=1, M(t)=\gamma(-t)^{1 / 2}$, and $K(t)=1+\left(\int_{-t}^{0} g(\theta) d \theta\right)^{1 / 2}$, for $t \geq 0$ (see [19, Theorem 1.3.8] for details).

For additional details concerning phase space we refer the reader to [19].

To obtain our results, we assume that the integrodifferential abstract Cauchy problem

$$
\begin{gathered}
D_{t}^{\alpha} x(t)=A x(t)+\int_{0}^{t} B(t-s) x(s) d s, \quad \alpha \in(1,2), \\
x(0)=z \in X, \quad x^{\prime}(0)=0
\end{gathered}
$$

has an associated $\alpha$-resolvent operator of bounded linear operators $\left(\mathcal{R}_{\alpha}(t)\right)_{t \geq 0}$ on $X$.

Definition 2.2. A one parameter family of bounded linear operators $\left(\mathcal{R}_{\alpha}(t)\right)_{t \geq 0}$ on $X$ is called a $\alpha$-resolvent operator of (2.3)-(2.4) if the following conditions are verified.

(a) The function $\mathcal{R}_{\alpha}(\cdot):[0, \infty) \rightarrow \mathcal{L}(X)$ is strongly continuous and $\mathcal{R}_{\alpha}(0) x=x$ for all $x \in X$ and $\alpha \in(1,2)$. 
(b) For $x \in D(A), \mathcal{R}_{\alpha}(\cdot) x \in C([0, \infty),[D(A)]) \cap C^{1}((0, \infty), X)$, and

$$
\begin{aligned}
& D_{t}^{\alpha} \mathcal{R}_{\alpha}(t) x=A \mathcal{R}_{\alpha}(t) x+\int_{0}^{t} B(t-s) \mathcal{R}_{\alpha}(s) x d s, \\
& D_{t}^{\alpha} \mathcal{R}_{\alpha}(t) x=\mathcal{R}_{\alpha}(t) A x+\int_{0}^{t} \mathcal{R}_{\alpha}(t-s) B(s) x d s,
\end{aligned}
$$

for every $t \geq 0$.

The existence of a resolvent operator for problem (2.3)-(2.4) was studied in [20]. In this paper, we have considered the following conditions.

(P1) The operator $A: D(A) \subseteq X \rightarrow X$ is a closed linear operator with $[D(A)]$ dense in $X$. There is positive constants $C_{0}=C_{0}(\phi)$, such that

$$
\Sigma_{0, \alpha \vartheta}=\{\lambda \in \mathbb{C}: \lambda \neq 0,|\arg (\lambda)|<\alpha \vartheta\} \subset \rho(A),
$$

where $\alpha \in(1,2), \vartheta=\phi+\pi / 2, \phi<\phi_{0}$ for some $\phi_{0} \in(0, \pi / 2]$, and $\|R(\lambda, A)\| \leq C_{0} /|\lambda|$ for all $\lambda \in \Sigma_{0, \alpha \vartheta}$.

(P2) For all $t \geq 0, B(t): D(B(t)) \subseteq X \rightarrow X$ is a closed linear operator, $D(A) \subseteq D(B(t))$, and $B(\cdot) x$ is strongly measurable on $(0, \infty)$ for each $x \in D(A)$. There exists $b(\cdot) \in$ $L_{\text {loc }}^{1}\left(\mathbb{R}^{+}\right)$(the notation $L_{\text {loc }}^{1}\left(\mathbb{R}^{+}\right)$stands for the set of all locally integrable functions from $\mathbb{R}^{+}$into $\mathbb{R}^{+}$) such that $\widehat{b}(\lambda)$ exists for $\operatorname{Re}(\lambda)>0$ and $\|B(t) x\| \leq b(t)\|x\|_{1}$ for all $t>0$ and $x \in D(A)$. Moreover, the operator-valued function $\widehat{B}: \Sigma_{0, \pi / 2} \rightarrow$ $\mathcal{L}([D(A)], X)$ has an analytical extension (still denoted by $\widehat{B})$ to $\Sigma_{0, \vartheta}=\{\lambda \in \mathbb{C}$ : $\lambda \neq 0,|\arg (\lambda)|<\vartheta\}$ such that $\|\widehat{B}(\lambda) x\| \leq\|\widehat{B}(\lambda)\|\|x\|_{1}$ for all $x \in D(A)$, and $\|\widehat{B}(\lambda)\|=$ $O(1 /|\lambda|)$, as $|\lambda| \rightarrow \infty$.

(P3) There exists a subspace $D \subseteq D(A)$ dense in $[D(A)]$ and positive constant $C_{1}$, such that $A(D) \subseteq D(A), \widehat{B}(\lambda)(D) \subseteq D(A),\|A \widehat{B}(\lambda) x\| \leq C_{1}\|x\|$ for every $x \in D$ and all $\lambda \in \Sigma_{\omega, \vartheta}$.

In the sequel, for $r>0$ and $\theta \in(\pi / 2, \vartheta)$,

$$
\Sigma_{r, \theta}=\{\lambda \in \mathbb{C}: \lambda \neq 0,|\lambda|>r,|\arg (\lambda)|<\theta\},
$$

for $\Gamma_{r, \theta}, \Gamma_{r, \theta}^{i}, i=1,2,3$, are the paths

$$
\begin{gathered}
\Gamma_{r, \theta}^{1}=\left\{t e^{i \theta}: t \geq r\right\}, \\
\Gamma_{r, \theta}^{2}=\left\{r e^{i \xi}:-\theta \leq \xi \leq \theta\right\}, \\
\Gamma_{r, \theta}^{3}=\left\{t e^{-i \theta}: t \geq r\right\},
\end{gathered}
$$


and $\Gamma_{r, \theta}=\bigcup_{i=1}^{3} \Gamma_{r, \theta}^{i}$ oriented counterclockwise. In addition, $\rho_{\alpha}\left(G_{\alpha}\right)$ are the sets

$$
\rho_{\alpha}\left(G_{\alpha}\right)=\left\{\lambda \in \mathbb{C}: G_{\alpha}(\lambda):=\lambda^{\alpha-1}\left(\lambda^{\alpha} I-A-\widehat{B}(\lambda)\right)^{-1} \in \mathcal{L}(X)\right\}
$$

We now define the operator family $\left(\mathcal{R}_{\alpha}(t)\right)_{t \geq 0}$ by

$$
\mathcal{R}_{\alpha}(t)= \begin{cases}\frac{1}{2 \pi i} \int_{\omega+\Gamma_{r, \theta}} e^{\lambda t} G_{\alpha}(\lambda) d \lambda, & t>0 \\ I, & t=0 .\end{cases}
$$

The following result has been established in [20, Theorem 2.1].

Theorem 2.3. Assume that conditions (P1)-(P3) are fulfilled, then there is a unique a-resolvent operator for problem (2.3)-(2.4).

In what follows, we always assume that the conditions (P1)-(P3) are verified.

We consider now the nonhomogeneous problem.

In the rest of this section, we discuss existence and regularity of solutions of

$$
\begin{gathered}
D_{t}^{\alpha} x(t)=A x(t)+\int_{0}^{t} B(t-s) x(s) d s+f(t), \quad t \in[0, a] \\
x(0)=z \in X, \quad x^{\prime}(0)=0
\end{gathered}
$$

where $\alpha \in(1,2)$ and $f \in L^{1}([0, a], X)$. In the sequel, $\mathcal{R}_{\alpha}(\cdot)$ is the operator function defined by (2.11). We begin by introducing the following concept of classical solution.

Definition 2.4. A function $x:[0, a] \rightarrow X, 0<a$ is called a classical solution of (2.12)-(2.13) on $[0, a]$ if $x \in C([0, a],[D(A)]) \cap C([0, a], X), g_{n-\alpha} * x:=\int_{0}^{t} g_{n-\alpha}(t-s) x d s \in C^{1}([0, a], X), n=1,2$; the condition (2.13) holds and (2.12) is verified on $[0, a]$.

Definition 2.5. Let $\alpha \in(1,2)$, we define the family $\left(\mathcal{S}_{\alpha}(t)\right)_{t \geq 0}$ by

$$
\mathcal{S}_{\alpha}(t) x:=\int_{0}^{t} g_{\alpha-1}(t-s) \mathcal{R}_{\alpha}(s) x d s, \quad x \in X
$$

for each $t \geq 0$.

The proof of the next result is in [20]. For reader's convenience, we will give the proof.

Lemma 2.6. If the function $\mathcal{R}_{\alpha}(\cdot)$ is exponentially bounded in $\mathcal{L}(X)$, then $\mathcal{S}_{\alpha}(\cdot)$ is exponentially bounded in $\mathcal{L}(X)$. 
Proof. If there are constants, $M, \omega>0$ such that $\left\|\mathcal{R}_{\alpha}(t)\right\| \leq M e^{\omega t}$, we obtain

$$
\begin{aligned}
\left\|\mathcal{S}_{\alpha}(t)\right\| & \leq \int_{0}^{t} g_{\alpha-1}(t-s)\left\|\mathcal{R}_{\alpha}(s)\right\| d s \leq \frac{M}{\Gamma(\alpha-1)} \int_{0}^{t}(t-s)^{\alpha-2} e^{\omega s} d s \\
& \leq \frac{M}{\Gamma(\alpha-1)} \int_{0}^{t} s^{\alpha-2} e^{\omega(t-s)} d s \leq \frac{M e^{\omega t}}{\Gamma(\alpha-1)} \int_{0}^{t} s^{\alpha-2} e^{-\omega s} d s \\
& \leq \frac{M e^{\omega t}}{\Gamma(\alpha-1) \omega^{\alpha-1}} \int_{0}^{t} s^{\alpha-2} e^{-s} d s \leq \frac{M e^{\omega t}}{\Gamma(\alpha-1) \omega^{\alpha-1}} \Gamma(\alpha-1) \\
& \leq M_{\omega} e^{\omega t}
\end{aligned}
$$

where $M_{\omega}=M / \omega^{\alpha-1}$.

The next result follows from Lemma 2.6. We will omit the proof.

Lemma 2.7. If the function $\mathcal{R}_{\alpha}(\cdot)$ is exponentially bounded in $\mathcal{L}([D(A)])$, then $\mathcal{S}_{\alpha}(\cdot)$ is exponentially bounded in $\mathcal{L}([D(A)])$.

We now establish a variation of constants formula for the solutions of (2.12)-(2.13). The proof of the next result is in [20]. For reader's convenience, we will give the proof.

Theorem 2.8. Let $z \in D(A)$. Assume that $f \in C([0, a], X)$ and $u(\cdot)$ is a classical solution of (2.12)(2.13) on $[0, a]$, then

$$
u(t)=\mathcal{R}_{\alpha}(t) z+\int_{0}^{t} \mathcal{S}_{\alpha}(t-s) f(s) d s, \quad t \in[0, a]
$$

Proof. The Cauchy problem (2.12)-(2.13) is equivalent to the Volterra equation

$$
u(t)=x+\int_{0}^{t} g_{\alpha}(t-s) A u(s) d s+\int_{0}^{t} g_{\alpha}(t-s) \int_{0}^{s} B(s-\xi) u(\xi) d \xi d s+\int_{0}^{t} g_{\alpha}(t-s) f(s) d s,
$$

and the $\alpha$-resolvent equation (2.6) is equivalent to

$$
\mathcal{R}_{\alpha}(t) x=x+\int_{0}^{t} g_{\alpha}(t-s) \mathcal{R}_{\alpha}(s) A x d s+\int_{0}^{t} g_{\alpha}(t-s) \int_{0}^{s} \mathcal{R}_{\alpha}(s-\xi) B(\xi) x d \xi d s
$$

To prove (2.16), we notice that

$$
\begin{aligned}
I * u & =\left(\mathcal{R}_{\alpha}-g_{\alpha} * \mathcal{R}_{\alpha} A-g_{\alpha} * \mathcal{R}_{\alpha} * B\right) * u \\
& =\mathcal{R}_{\alpha} *\left(u-g_{\alpha} A u-g_{\alpha} * B * u\right) \\
& =\mathcal{R}_{\alpha} *\left(I+g_{\alpha} * f\right) \\
& =\mathcal{R}_{\alpha} * I+g_{\alpha} * \mathcal{R}_{\alpha} * f \\
& =\mathcal{R}_{\alpha} * I+g_{1} * g_{\alpha-1} * \mathcal{R}_{\alpha} * f .
\end{aligned}
$$


Therefore,

$$
\begin{aligned}
\int_{0}^{t} u(s) d s & =\int_{0}^{t} \mathcal{R}_{\alpha}(s) x d s+\int_{0}^{t} \int_{0}^{s} g_{\alpha-1}(s-\xi) \int_{0}^{\xi} \mathcal{R}_{\alpha}(\xi-u) f(u) d u d \xi d s \\
& =\int_{0}^{t} \mathcal{R}_{\alpha}(s) x d s+\int_{0}^{s} \int_{0}^{s} \int_{u}^{s} g_{\alpha-1}(s-\xi) \mathcal{R}_{\alpha}(\xi-u) f(u) d u d \xi d s \\
& =\int_{0}^{t} \mathcal{R}_{\alpha}(s) x d s+\int_{0}^{t} \int_{0}^{s} \int_{0}^{s-u} g_{\alpha-1}(s-u-\xi) \mathcal{R}_{\alpha}(\xi) f(u) d \xi d u d s \\
& =\int_{0}^{t} \mathcal{R}_{\alpha}(s) x d s+\int_{0}^{t} \int_{0}^{s} \int_{0}^{s-u} g_{\alpha-1}(s-u-\xi) \mathcal{R}_{\alpha}(\xi) f(u) d \xi d u d s \\
& =\int_{0}^{t} \mathcal{R}_{\alpha}(s) x d s+\int_{0}^{t} \int_{0}^{s} S_{\alpha}(s-u) f(u) d u d s .
\end{aligned}
$$

We obtain

$$
u(t)=\mathcal{R}_{\alpha}(t) x+\int_{0}^{t} \mathcal{S}_{\alpha}(t-u) f(u) d u
$$

It is clear from the preceding definition that $R_{\alpha}(\cdot) z$ is a solution of problem (2.3)-(2.4) on $(0, \infty)$ for $z \in D(A)$.

Definition 2.9. Let $f \in L^{1}([0, a], X)$. A function $u \in C([0, a], X)$ is called a mild solution of (2.12)-(2.13) if

$$
u(t)=\mathcal{R}_{\alpha}(t) z+\int_{0}^{t} \mathcal{S}_{\alpha}(t-s) f(s) d s, \quad t \in[0, a], z \in X
$$

The proof of the next result is in [20]. For reader's convenience, we will give the proof.

Theorem 2.10. Let $z \in D(A)$ and $f \in C([0, a], X)$. If $f \in L^{1}([0, a],[D(A)])$, then the mild solution of (2.12)-(2.13) is a classical solution.

Proof. To begin with, we study the case in which $f \in C^{1}([0, a],[D(A)])$. Let $w$ be the mild solution of (2.12)-(2.13) and assume that $z=0$. It is easy to see that $w \in C([0, a],[D(A)])$ and

$$
\|w\|_{C([0, a],[D(A)])} \leq M\|f\|_{L^{1}([0, a]:[D(A)])}
$$

where $M$ is given by $M_{\omega} e^{\omega a}$. From [7, Lemma 3.12], we obtain that $w(\cdot)$ is a classical solution and satisfies

$$
D_{t}^{\alpha} w(t)=A w(t)+\int_{0}^{t} B(t-s) w(s) d s+f(t)
$$


Moreover, from (2.24) and taking into account that $\|B(t) x\| \leq b(t)\|x\|_{1}$ for all $t>0$ and $x \in D(A)$, we deduce the existence of constants $C_{1}, C_{2}>0$ (which are independent from $f$ ) such that

$$
\|w\|_{C([0, a],[D(A)])}+\left\|D_{t}^{\alpha} w\right\|_{C([0, a], X)} \leq C_{1}\|f\|_{L^{1}([0, a]:[D(A)])}+C_{2}\|f\|_{C([0, a], X)} .
$$

Now, we assume that $f \in L^{1}([0, a]:[D(A)])$. Let $\left(f_{n}\right)_{n \in \mathbb{N}}$ be a sequence in $C^{1}([0, a],[D(A)])$ such that $f_{n} \rightarrow f$ in $L^{1}([0, a],[D(A)]) \cap C([0, a], X)$. From [7, Lemma 3.12], we know that $w_{n}=\mathcal{S}_{\alpha} * f_{n}, n \in \mathbb{N}$, is a classical solution of (2.12)-(2.13) with $f_{n}$ in the place of $f$. By using the estimate (2.25), we deduce the existence of functions $w, v \in C([0, a], X)$, such that $w_{n} \rightarrow w=\mathcal{S}_{\alpha} * f$ in $C([0, a],[D(A)])$ and $D_{t}^{\alpha} w_{n} \rightarrow v$ in $C([0, a], X)$. These facts, jointly with our assumptions on $B(\cdot)$, permit to conclude that $B * w_{n} \rightarrow B * w$ in $C([0, a], X)$. On the other hand,

$$
\begin{aligned}
J_{t}^{\alpha} v(t) & =\lim _{n \rightarrow \infty} J_{t}^{\alpha} D_{t}^{\alpha} w_{n}(t) \\
& =\lim _{n \rightarrow \infty}\left(w_{n}(t)-\sum_{k=0}^{1} w_{n}^{(k)}(0) g_{k+1}(t)\right) \\
& =\lim _{n \rightarrow \infty}\left(w_{n}(t)-w_{n}(0)-w_{n}^{\prime}(0) t\right) \\
& =w(t)-w^{\prime}(0) t
\end{aligned}
$$

we obtain

$$
v=D_{t}^{\alpha} J_{t}^{\alpha} v=D_{t}^{\alpha} w(t)
$$

Now, by making $n \rightarrow \infty$ on

$$
D_{t}^{\alpha} w_{n}(t)=A w_{n}(t)+\int_{0}^{t} B(t-s) w_{n}(s) d s+f_{n}(t), \quad t \in[0, a]
$$

we conclude that $w=\mathcal{S}_{\alpha} * f$ is a classical solution of (2.12)-(2.13). The proof is finished.

The proof of the next result is in [20]. For reader's convenience, we will give the proof.

Theorem 2.11. Let $z \in D(A)$ and $f \in C([0, a], X)$. If $f \in W^{1,1}([0, a], X)$, then the mild solution of (2.12)-(2.13) is a classical solution.

Proof. Let $f \in W^{1,1}([0, a], X)$, there is $\left(f_{n}\right)_{n \in \mathbb{N}}$ on $C^{1}([0, a],[D(A)])$ such that $f_{n} \rightarrow f$ on $C([0, a], X)$ and $f_{n} \rightarrow f$ on $W^{1,1}([0, a]: X)$. Put $w_{n}(t)=\mathcal{S}_{\alpha} * f_{n}$ proceeding as in the proof 
of Theorem 2.10. It follows that $w_{n}(\cdot)$ is a classical solution of (2.12)-(2.13). Moreover, from [7, Lemma 3.13], we obtain

$$
\begin{aligned}
-\mho_{\alpha}(t) f(0) & =\int_{0}^{t} \frac{d}{d s}\left[\mho_{\alpha}(t-s) f(s)\right] d s \\
& =-\int_{0}^{t} S_{\alpha}(t-s) f(s) d s+\int_{0}^{t} \mho_{\alpha}(t-s) f^{\prime}(s) d s, \quad t \in[0, a] \\
w(t) & =\mho_{\alpha}(t) f(0)+\int_{0}^{t} \mho_{\alpha}(t-s) f^{\prime}(s) d s, \quad t \in[0, a]
\end{aligned}
$$

from which we deduce the existence of positive constants $C_{3}, C_{4}$ (independent from $f$ ) such that

$$
\|w\|_{C([0, a],[D(A)])}+\left\|D_{t}^{\alpha} w\right\|_{C([0, a], X)} \leq C_{3}\|f\|_{C([0, a], X)}+C_{4}\left\|f^{\prime}\right\|_{L^{1}([0, a], X)} .
$$

With similar arguments as in the proof of Theorem 2.10, we conclude that $w=\mathcal{S}_{\alpha} * f$ is a classical solution of (2.12)-(2.13). We omit additional details. The proof is completed.

To establish our existence results, we need the following Lemma.

Lemma 2.12. Let $\alpha \in(1,2)$. If $R\left(\lambda_{0}^{\alpha}, A\right):=\left(\lambda_{0}^{\alpha}-A\right)^{-1}$ is compact for some $\lambda_{0}^{\alpha} \in \rho(A)$, then $\boldsymbol{R}_{\alpha}(t)$ and $\mathcal{S}_{\alpha}(t)$ are compact for all $t>0$.

Proof. From the resolvent identity, it follows that $R\left(\lambda^{\alpha}, A\right)$ is compact for every $\lambda^{\alpha} \in \rho(A)$. We have from [20, Lemma 2.2] that $G_{\alpha}(\lambda)=\lambda^{\alpha-1} R\left(\lambda^{\alpha}, A\right)\left[I-\widehat{B}(\lambda) R\left(\lambda^{\alpha}, A\right)\right]^{-1}$ is a compact operator for $\lambda \in \Sigma_{r, \theta}$; therefore, $\mathcal{R}_{\alpha}(t)$ is a compact operator for $t>0$.

From [20, Lemma 2.5], we have, $\mathcal{R}_{\alpha}(t)$ is uniformly continuous for $0<t<a$, for any $a$ fixed, we can select points $0=s_{0}<s_{1}<\cdots<s_{n}=t$, such that if $\left|s_{i}-s_{i+1}\right|<\delta$, we obtain $\left\|\mathcal{R}_{\alpha}(s)-\mathcal{R}_{\alpha}\left(s_{i}\right)\right\|_{\mathcal{L}(X)}<\varepsilon$, for all $s \in\left[s_{i}, s_{i+1}\right], i=1,2, \ldots, n-1$ and $\left\|\int_{0}^{s_{1}} g_{\alpha-1}(t-s) \mathcal{R}_{\alpha}(s) d s\right\|_{\mathcal{L}(X)}<$ $\varepsilon$.

Therefore, for all $x \in B_{r}(0, X)$, we have that

$$
\begin{aligned}
\mathcal{S}_{\alpha}(t) x= & \int_{0}^{t} g_{\alpha-1}(t-s) \mathcal{R}_{\alpha}(s) x d s \\
= & \int_{0}^{s_{1}} g_{\alpha-1}(t-s) \mathcal{R}_{\alpha}(s) x d s+\sum_{i=1}^{n-1} \int_{s_{i}}^{s_{i}+1} g_{\alpha-1}(t-s)\left[\mathcal{R}_{\alpha}(s) x-\mathcal{R}_{\alpha}\left(s_{i}\right) x\right] d s \\
& +\sum_{i=1}^{n-1} \mathcal{R}_{\alpha}\left(s_{i}\right) \int_{s_{i}}^{s_{i}+1} g_{\alpha-1}(t-s) x d s .
\end{aligned}
$$

Noting now that

$$
\left\|\sum_{i=1}^{n-1} \int_{s_{i}}^{s_{i}+1} g_{\alpha-1}(t-s)\left[\mathcal{R}_{\alpha}(s) x-\mathcal{R}_{\alpha}\left(s_{i}\right) x\right] d s\right\| \leq \frac{\varepsilon a^{\alpha-1}}{\Gamma(\alpha)} r,
$$


from (2.31), we find that

$$
\mathcal{S}_{\alpha}(t) B_{r}(0, X) \subset B_{r \varepsilon}(0, X)+B_{\left(a^{\alpha-1} / \Gamma(\alpha)\right) \varepsilon r}(0, X)+\sum_{i=1}^{n-1} \mathcal{R}_{\alpha}\left(s_{i}\right) B_{\left(a^{\alpha-1} / \Gamma(\alpha)\right) \varepsilon r}(0, X)
$$

Thus,

$$
\mathcal{S}_{\alpha}(t) B_{r}(0, X) \subset C_{\varepsilon}+K_{\varepsilon}
$$

where $K_{\varepsilon}=\sum_{i=1}^{n-1} \mathcal{R}_{\alpha}\left(s_{i}\right) B_{\left(a^{\alpha-1} / \Gamma(\alpha)\right) \varepsilon r}(0, X)$ is compact and $C_{\varepsilon}=B_{r \varepsilon}(0, X)+B_{\left(a^{\alpha-1} / \Gamma(\alpha)\right) \varepsilon r}(0, X)$, then we observe that $\operatorname{diam}\left(C_{\varepsilon}\right) \rightarrow 0$ as $\varepsilon \rightarrow 0$. This permits us to conclude that $\mathcal{S}_{\alpha}(t) B_{r}(0, X)$ is relatively compact in $X$. This proves that $S_{\alpha}(t)$ is a compact operator for all $t>0$.

For completeness, we include the following well-known result.

Theorem 2.13 (Leray-Schauder alternative, [21, Theorem 6.5.4]). Let $D$ be a closed convex subset of a Banach space $Z$ with $0 \in D$. Let $G: D \rightarrow D$ be a completely continuous map. Then, $G$ has a fixed point in $D$ or the set $\{z \in D: z=\lambda G(z), 0<\lambda<1\}$ is unbounded.

\section{Existence Results}

In this section, we study the existence of mild solutions for system (1.1)-(1.2). Along this section, $M$ is a positive constant such that $\left\|\mathcal{R}_{\alpha}(t)\right\| \leq M$ and $\left\|\mathcal{S}_{\alpha}(t)\right\| \leq M$ for every $t \in I$. We adopt the notion of mild solutions for (1.1)-(1.2) from the one given in [20].

Definition 3.1. A function $u:(-\infty, b] \rightarrow X$ is called a mild solution of the neutral system (1.1)-(1.2) on $[0, b]$ if $u_{0}=\varphi, u_{\rho\left(t, u_{t}\right)} \in B, u_{[0, a]} \in C([0, b], X)$, and

$$
u(t)=\mathcal{R}_{\alpha}(t) \varphi(0)+\int_{0}^{t} \mathcal{S}_{\alpha}(t-s) f\left(s, u_{\rho\left(s, u_{s}\right)}\right) d s, \quad t \in[0, b]
$$

To prove our results, we always assume that $\rho: I \times \mathcal{B} \rightarrow(-\infty, b]$ is continuous and that $\varphi \in \mathcal{B}$. If $x \in C([0, b] ; X)$, we define $\bar{x}:(-\infty, b] \rightarrow X$ as the extension of $x$ to $(-\infty, b]$ such that $\bar{x}_{0}=\varphi$. We define $\tilde{x}:(-\infty, b] \rightarrow X$ such that $\tilde{x}=x+y$ where $y:(-\infty, b] \rightarrow X$ is the extension of $\varphi \in B$, such that $y(t)=\mathcal{R}_{\alpha}(t) \varphi(0)$ for $t \in I$.

In the sequel, we introduce the following conditions.

$\left(\mathrm{H}_{1}\right)$ The function $f:[0, b] \times \mathbb{B} \rightarrow X$ verifies the following conditions:

(i) the function $f(t, \cdot): \mathbb{B} \rightarrow X$ is continuous for every $t \in[0, b]$, and for every $\psi \in \mathbb{B}$, the function $f(\cdot, \psi):[0, b] \rightarrow X$ is strongly measurable,

(ii) there exist $m_{f} \in C([0, b],[0, \infty))$ and a continuous nondecreasing function $\Omega_{f}$ : $[0, \infty) \rightarrow(0, \infty)$, such that $\|f(t, \psi)\| \leq m_{f}(t) \Omega_{f}\left(\|\psi\|_{\mathcal{B}}\right)$, for all $(t, \psi) \in[0, b] \times \mathbb{B}$.

$\left(\mathrm{H}_{2}\right)$ For all $t, s \in[0, b], t \geq s$ and $r>0$, the set $\left\{f(s, \psi): s \in[0, t],\|\psi\|_{\mathcal{B}} \leq r\right\}$ is bounded in $X$. 
$\left(\mathrm{H}_{\varphi}\right)$ The function $t \rightarrow \varphi_{t}$ is well defined and continuous from the set

$$
\mathcal{R}\left(\rho^{-}\right)=\{\rho(s, \psi):(s, \psi) \in I \times \mathbb{B}, \rho(s, \psi) \leq 0\}
$$

into $\mathbb{B}$, and there exists a continuous and bounded function $J^{\varphi}: \mathcal{R}\left(\rho^{-}\right) \rightarrow(0, \infty)$, such that $\left\|\varphi_{t}\right\|_{\mathcal{B}} \leq J^{\varphi}(\mathrm{t})\|\varphi\|_{\mathcal{B}}$ for every $t \in \mathcal{R}(\rho)$.

Remark 3.2. The condition $\left(\mathrm{H}_{\varphi}\right)$ is frequently verified by continuous and bounded functions.

Remark 3.3. In the rest of this section, $M_{b}$ and $K_{b}$ are the constants $M_{b}=\sup _{s \in[0, b]} M(s)$ and $K_{b}=\sup _{s \in[0, b]} K(s)$.

Lemma 3.4 (see [13, Lemma 2.1]). Let $x:(-\infty, b] \rightarrow X$ be continuous on $[0, b]$ and $x_{0}=\varphi$. If $\left(\mathrm{H}_{\varphi}\right)$ holds, then

$$
\left\|x_{s}\right\|_{\mathcal{B}} \leq\left(M_{b}+J^{\varphi}\right)\|\varphi\|_{\mathcal{B}}+K_{b} \sup \{\|x(\theta)\| ; \theta \in[0, \max \{0, s\}]\},
$$

$s \in \mathcal{R}\left(\rho^{-}\right) \cup I$, where $J^{\varphi}=\sup _{t \in \mathcal{R}\left(\rho^{-}\right)} J^{\varphi}(t)$.

Theorem 3.5. Let conditions $\left(H_{1}\right),\left(H_{2}\right)$, and $\left(H_{\varphi}\right)$ hold, and assume that $\mathcal{R}(\cdot) \in C((0, b] ; \mathcal{L}(X))$. If $M K_{b} \lim \inf _{\xi \rightarrow \infty}\left(\Omega_{f}(\xi) / \xi\right) \int_{0}^{b} m_{f}(s) d s<1$, then there exists a mild solution of (1.1)-(1.2) on $[0, b]$.

Proof. Let $\bar{\varphi}:(-\infty, b] \rightarrow X$ be the extension of $\varphi$ to $(-\infty, b]$ such that $\bar{\varphi}(\theta)=\varphi(0)$ on $I=$ $[0, b]$. Consider the space $S(b)=\{u \in C(I ; X): u(0)=\varphi(0)\}$ endowed with the uniform convergence topology and define the operator $\Gamma: S(b) \rightarrow S(b)$ by

$$
\Gamma x(t)=\mathcal{R}_{\alpha}(t) \varphi(0)+\int_{0}^{t} \mathcal{S}_{\alpha}(t-s) f\left(s, \bar{x}_{\rho\left(s, \bar{x}_{s}\right)}\right) d s,
$$

for $t \in[0, b]$. It is easy to see that $\Gamma S(b) \subset S(b)$. We prove that there exists $r>0$ such that $\Gamma\left(B_{r}\left(\left.\bar{\varphi}\right|_{I}, S(b)\right)\right) \subseteq B_{r}\left(\left.\bar{\varphi}\right|_{I}, S(b)\right)$. If this property is false, then for every $r>0$ there exist $x^{r} \in$ $B_{r}\left(\left.\bar{\varphi}\right|_{I}, S(b)\right)$ and $t^{r} \in I$ such that $r<\left\|\Gamma x^{r}\left(t^{r}\right)-\varphi(0)\right\|$. Then, from Lemma 3.4, we find that

$$
\begin{aligned}
r \leq & \left\|\Gamma x^{r}\left(t^{r}\right)-\phi(0)\right\| \leq(M+1) H\|\varphi\|_{\mathcal{B}} \\
& +M \Omega_{f}\left(\left(M_{b}+J^{\varphi}\right)\|\varphi\|_{b}+K_{b}(r+\|\varphi(0)\|)\right) \int_{0}^{b} m_{f}(s) d s,
\end{aligned}
$$

and hence,

$$
1 \leq M K_{b} \liminf _{\xi \rightarrow \infty} \frac{\Omega_{f}(\xi)}{\xi} \int_{0}^{b} m_{f}(s) d s,
$$

which contradicts our assumption. 
Let $r>0$ be such that $\Gamma\left(B_{r}\left(\left.\bar{\varphi}\right|_{I}, S(b)\right)\right) \subseteq B_{r}\left(\left.\bar{\varphi}\right|_{I}, S(b)\right)$, in the sequel; $r^{*}$ and $r^{* *}$ are the numbers defined by $r^{*}:=\left(M_{b}+J^{\varphi}\right)\|\varphi\|_{\mathcal{B}}+K_{b}(r+\|\varphi(0)\|)$ and $r^{* *}:=\Omega_{g}\left(r^{*}\right) \int_{0}^{b} m_{g}(s) d s$. To prove that $\Gamma$ is a condensing operator, we introduce the decomposition $\Gamma=\Gamma_{1}+\Gamma_{2}$, where

$$
\begin{gathered}
\Gamma_{1} x(t)=\mathcal{R}_{\alpha}(t) \varphi(0), \\
\Gamma_{2} x(t)=\int_{0}^{t} \mathcal{S}_{\alpha}(t-s) f\left(s, \bar{x}_{\rho\left(s, \bar{x}_{s}\right)}\right) d s,
\end{gathered}
$$

for $t \in I$.

It is easy to see that $\Gamma_{1}(\cdot)$ is continuous and a contraction on $B_{r}\left(\left.\bar{\varphi}\right|_{I}, S(b)\right)$. Next, we prove that $\Gamma_{2}(\cdot)$ is completely continuous from $B_{r}\left(\left.\bar{\varphi}\right|_{I}, S(b)\right)$ into $B_{r}\left(\left.\bar{\varphi}\right|_{I}, S(b)\right)$.

Step 1. Let $x \in B_{r}\left(\left.\bar{\varphi}\right|_{I}, S(b)\right)$, and let $\epsilon$ be a positive real number such that $0<\epsilon<t \leq b$. We can infer that

$$
\begin{aligned}
\Gamma_{2} x(t) & =\int_{0}^{t-\epsilon} \mathcal{S}_{\alpha}(t-s) f\left(s, \bar{x}_{\rho\left(s, \bar{x}_{s}\right)}\right) d s+\int_{t-\epsilon}^{t} \mathcal{S}_{\alpha}(t-s) f\left(s, \bar{x}_{\rho\left(s, \bar{x}_{s}\right)}\right) d s \\
& \in(t-\epsilon) \overline{\operatorname{co}(K)}+C_{\epsilon}
\end{aligned}
$$

where $K=\left\{S_{\alpha}(t-\theta) f(\theta, \psi): \theta \in[0, t-\epsilon],\|\psi\|_{\mathcal{B}} \leq r^{*}\right\}, \operatorname{co}(K)$ is the convex hull of the set $K$ and $C_{\epsilon}=\left\{\int_{t-\epsilon}^{t} \mathcal{S}_{\alpha}(t-s) f\left(s, \bar{x}_{\rho\left(s, \bar{x}_{s}\right)}\right) d s: x \in B_{r}\left(\left.\bar{\varphi}\right|_{I}, S(b)\right)\right\}$, since

$$
\operatorname{diam} C_{\epsilon} \leq 2 M \Omega_{f}\left(r^{*}\right) \int_{t-\epsilon}^{t} m_{f}(s) d s,
$$

which proves that $\Gamma_{2}\left(B_{r}\left(\left.\bar{\varphi}\right|_{I}, S(b)\right)\right)(t)$ is relatively compact in $X$.

Step 2. The set $\Gamma_{2}\left(B_{r}\left(\left.\bar{\varphi}\right|_{I}, S(b)\right)\right)$ is equicontinuous on $[0, b]$.

Let $0<\epsilon<t<b$ and $0<\delta<\epsilon$ such that $\left\|\mathcal{S}_{\alpha}(s)-\mathcal{S}_{\alpha}\left(s^{\prime}\right)\right\| \leq \epsilon$ for every $s, s^{\prime} \in[\epsilon, b]$ with $\left|s-s^{\prime}\right| \leq \delta$. Under these conditions, for $x \in B_{r}\left(\left.\bar{\varphi}\right|_{I}, S(b)\right)$ and $0<h \leq \delta$ with $t+h \in[0, b]$, we get

$$
\begin{aligned}
&\left\|\Gamma_{2} x(t+h)-\Gamma_{2} x(t)\right\| \\
& \leq \int_{0}^{t-\epsilon}\left\|\left[\mathcal{S}_{\alpha}(t+h-s)-\mathcal{S}_{\alpha}(t-s)\right] f\left(s, \bar{x}_{\rho\left(s, \bar{x}_{s}\right)}\right)\right\| d s \\
& \quad+\int_{t-\epsilon}^{t}\left\|\left[\mathcal{S}_{\alpha}(t+h-s)-\mathcal{S}_{\alpha}(t-s)\right] f\left(s, \bar{x}_{\rho\left(s, \bar{x}_{s}\right)}\right)\right\| d s \\
& \quad+\int_{t}^{t+h}\left\|\mathcal{S}_{\alpha}(t+h-s) f\left(s, \bar{x}_{\rho\left(s, \bar{x}_{s}\right)}\right)\right\| d s \\
& \leq \epsilon \Omega_{f}\left(r^{*}\right) \int_{0}^{b} m_{f}(s) d s+2 M \Omega_{f}\left(r^{*}\right) \int_{t-\epsilon}^{t} m_{f}(s) d s+M \Omega_{f}\left(r^{*}\right) \int_{t}^{t+h} m_{f}(s) d s,
\end{aligned}
$$


which shows that the set of functions $\Gamma_{2}\left(B_{r}\left(\left.\bar{\varphi}\right|_{I}, S(b)\right)\right)$ is right equicontinuity at $t \in(0, b)$. A similar procedure permits us to prove the right equicontinuity at zero and the left equicontinuity at $t \in(0, b]$. Thus, $\Gamma_{2}\left(B_{r}\left(\left.\bar{\varphi}\right|_{I}, S(b)\right)\right)$ is equicontinuous. By using a similar procedure to proof of the [13, Theorem 2.3], we prove that that $\Gamma_{2}(\cdot)$ is continuous on $B_{r}\left(\left.\bar{\varphi}\right|_{I}, S(b)\right)$, which completes the proof $\Gamma_{2}(\cdot)$ is completely continuous.

The existence of a mild solution for (1.1)-(1.2) is now a consequence of [22, Theorem 4.3.2]. This completes the proof.

Theorem 3.6. Let conditions $\left(H_{1}\right),\left(H_{2}\right),\left(H_{\varphi}\right)$ hold, $\rho(t, \psi) \leq t$ for every $(t, \psi) \in I \times \mathbb{B}$, and assume that $R_{\alpha}(\cdot) \in C((0, b] ; \mathcal{L}(X))$. If

$$
K_{b} M \int_{0}^{b} m_{f}(s) d s<\int_{C}^{\infty} \frac{1}{\Omega_{f}(s)} d s
$$

where $C=\left(M_{b}+K_{b} M H+J^{\varphi}\right)\|\varphi\|_{B}$, then there exists a mild solution of $(1.1)-(1.2)$ on $[0, b]$.

Proof. Let $\Gamma: C([0, b] ; X) \rightarrow C([0, b] ; X)$ be the operator defined by (3.4). In the sequel we use Theorem 2.13. If $x^{\lambda}=\lambda \Gamma x^{\lambda}, \lambda \in(0,1)$, then from Lemma 3.4, we have that

$$
\left\|x^{\lambda}(t)\right\| \leq M H\|\varphi\|_{\mathcal{B}}+\int_{0}^{t} M m_{f}(s) \Omega_{f}\left(\left(M_{b}+J^{\varphi}\right)\|\varphi\|_{\mathcal{B}}+K_{b}\left\|x^{\lambda}\right\|_{s}\right) d s,
$$

since $\rho\left(s, \bar{x}_{s}^{\lambda}\right) \leq s$ for every $s \in[0, b]$. If $\alpha^{\lambda}(t)=\left(M_{b}+J^{\varphi}\right)\|\varphi\|_{\mathcal{B}}+K_{b}\left\|x^{\lambda}\right\|_{t}$, we obtain that

$$
\alpha^{\lambda}(t) \leq\left(K_{b} M H+M_{b}+J^{\varphi}\right)\|\varphi\|_{\mathcal{B}}+K_{b} M \int_{0}^{t} m_{f}(s) \Omega_{f}\left(\alpha^{\lambda}(s)\right) d s
$$

Denoting by $\beta_{\lambda}(t)$ the right-hand side of the last inequality, we obtain that

$$
\beta_{\lambda}^{\prime}(t) \leq K_{b} M m_{f}(t) \Omega_{f}\left(\beta_{\lambda}(t)\right)
$$

and hence

$$
\int_{C}^{\beta_{\lambda}(t)} \frac{1}{\Omega_{f}(s)} d s \leq K_{b} M \int_{0}^{b} m_{f}(s) d s
$$

This inequality and (3.11) permit us to conclude that the set of functions $\left\{\beta_{\lambda}: \lambda \in(0,1)\right\}$ is bounded, which in turn shows that $\left\{x^{\lambda}: \lambda \in(0,1)\right\}$ is bounded.

By using a similar procedure allows to proof Theorem 3.5, we obtain that $\Gamma$ is completely continuous. By Theorem 2.13, the proof is ended. 


\section{Example}

To finish this section, we apply our results to study an integrodifferential equation which arises in the theory of heat equation. Consider the system

$$
\begin{gathered}
\frac{\partial^{\alpha}}{\partial t^{\alpha}} u(t, \xi)=\frac{\partial^{2} u(t, \xi)}{\partial \xi^{2}}+\int_{0}^{t}(t-s)^{\delta} e^{-\gamma(t-s)} \frac{\partial^{2} u(s, \xi)}{\partial \xi^{2}} d s \\
+\int_{-\infty}^{t} a(s-t) u\left(s-\rho_{1}(t) \rho_{2}(\|u(t)\|), \xi\right) d s, \quad(t, \xi) \in I \times[0, \pi], \\
u(t, 0)=u(t, \pi)=0, \quad t \in[0, b], \\
u_{t}(0, \xi)=0, \quad \xi \in[0, \pi], \\
u(\theta, \xi)=\varphi(\theta, \xi), \quad \theta \leq 0, \xi \in[0, \pi] .
\end{gathered}
$$

In this system, $\alpha \in(1,2), \delta, \gamma$ are positive numbers and $\partial^{\alpha} / \partial t^{\alpha}=D_{t}^{\alpha}$. To represent this system in the abstract form (1.1)-(1.2), we choose the spaces $X=L^{2}([0, \pi])$ and $\mathcal{B}=C_{0} \times L^{2}(g, X)$, see Example 2.1 for details. In the sequel, $A: D(A) \subseteq X \rightarrow X$ is the operator given by $A x=x^{\prime \prime}$ with domain $D(A)=\left\{x \in X: x^{\prime \prime} \in X, x(0)=x(\pi)=0\right\}$. It is well known that $\Delta x=x^{\prime \prime}$ is the infinitesimal generator of an analytic semigroup $(T(t))_{t>0}$ on $X$. Hence, $A$ is sectorial of type and (P1) is satisfied. We also consider the operator $B(t): D(A) \subseteq X \rightarrow X$, $t \geq 0, B(t) x=t^{\delta} e^{-\gamma t} \Delta x$ for $x \in D(A)$. Moreover, it is easy to see that conditions (P2)-(P3) in Section 2 are satisfied with $b(t)=t^{\delta} e^{-\gamma t}$ and $D=C_{0}^{\infty}([0, \pi])$, where $C_{0}^{\infty}([0, \pi])$ is the space of infinitely differentiable functions that vanish at $\xi=0$ and $\xi=\pi$.

We next consider the problem of the existence of mild solutions for the system (4.1). To this end, we introduce the following functions:

$$
f(t, \varphi)(\xi)=\int_{-\infty}^{0} a(-s) \varphi(s, \xi) d s, \quad \rho(s, \psi)=\rho_{1}(s) \rho_{2}(\|\varphi(0)\|) .
$$

Under the above conditions, we can represent the system (4.1) in the abstract form (2.12)(2.13). The following result is a direct consequence of Theorem 3.5.

Proposition 4.1. Let $\varphi \in ß$ be such that condition $\left(\mathrm{H}_{\varphi}\right)$ holds, the functions $\rho_{1}, \rho_{2}$ are bounded, and assume that the above conditions are fulfilled, then there exists a mild solution of $(4.1)$ on $[0, b]$.

\section{Acknowledgments}

The final version of this paper was finished while the third author was visiting the Universidade Federal de Pernambuco (Recife, Brasil) during Desember 2010-January 2011. The third author would like to thank the Functional Equations Group for their kind invitation and hospitality. The authors are grateful to the referees for pointing out omissions and misprints, and demanding vigorously details and clarifications. J. P. C. dos Santos is partially supported by FAPEMIG/Brazil under Grant no. CEX-APQ-00476-09. C. Cuevas is partially supported by CNPQ/Brazil under Grant no. 300365/2008-0. B. de Andrade is partially supported by CNPQ/Brazil under Grant no. 100994/2011-3. 


\section{References}

[1] M. Bartha, "Periodic solutions for differential equations with state-dependent delay and positive feedback," Nonlinear Analysis: Theory, Methods \& Applications, vol. 53, no. 6, pp. 839-857, 2003.

[2] Y. Cao, J. Fan, and T. C. Gard, "The effects of state-dependent time delay on a stage-structured population growth model," Nonlinear Analysis: Theory, Methods E Applications, vol. 19, no. 2, pp. 95$105,1992$.

[3] A. Domoshnitsky, M. Drakhlin, and E. Litsyn, "On equations with delay depending on solution," Nonlinear Analysis: Theory, Methods \& Applications, vol. 49, no. 5, pp. 689-701, 2002.

[4] F. Chen, D. Sun, and J. Shi, "Periodicity in a food-limited population model with toxicants and state dependent delays," Journal of Mathematical Analysis and Applications, vol. 288, no. 1, pp. 136-146, 2003.

[5] F. Hartung, "Linearized stability in periodic functional differential equations with state-dependent delays," Journal of Computational and Applied Mathematics, vol. 174, no. 2, pp. 201-211, 2005.

[6] F. Hartung, "Parameter estimation by quasilinearization in functional differential equations with state-dependent delays: a numerical study," Nonlinear Analysis: Theory, Methods \& Applications, vol. 47, no. 7, pp. 4557-4566, 2001.

[7] F. Hartung, T. L. Herdman, and J. Turi, "Parameter identification in classes of neutral differential equations with state-dependent delays," Nonlinear Analysis: Theory, Methods E Applications, vol. 39, no. 3, pp. 305-325, 2000.

[8] Y. Kuang and H. L. Smith, "Slowly oscillating periodic solutions of autonomous state-dependent delay equations," Nonlinear Analysis: Theory, Methods \& Applications, vol. 19, no. 9, pp. 855-872, 1992.

[9] R. Torrejón, "Positive almost periodic solutions of a state-dependent delay nonlinear integral equation," Nonlinear Analysis: Theory, Methods E Applications, vol. 20, no. 12, pp. 1383-1416, 1993.

[10] Y. Li, "Periodic solutions for delay Lotka-Volterra competition systems," Journal of Mathematical Analysis and Applications, vol. 246, no. 1, pp. 230-244, 2000.

[11] J. P. C. dos Santos, “On state-dependent delay partial neutral functional integro-differential equations," Applied Mathematics and Computation, vol. 216, no. 5, pp. 1637-1644, 2010.

[12] J. P. C. dos Santos, "Existence results for a partial neutral integro-differential equation with statedependent delay," Electronic Journal of Qualitative Theory of Differential Equations, vol. 2010, no. 29, pp. $1-12,2010$.

[13] E. Hernández, A. Prokopczyk, and L. Ladeira, "A note on partial functional differential equations with state-dependent delay," Nonlinear Analysis: Real World Applications, vol. 7, no. 4, pp. 510-519, 2006.

[14] E. Hernández M. and M. A. McKibben, "On state-dependent delay partial neutral functionaldifferential equations," Applied Mathematics and Computation, vol. 186, no. 1, pp. 294-301, 2007.

[15] E. Hernández Morales, M. A. McKibben, and H. R. Henríquez, "Existence results for partial neutral functional differential equations with state-dependent delay," Mathematical and Computer Modelling, vol. 49, no. 5-6, pp. 1260-1267, 2009.

[16] T. Maraaba, D. Baleanu, and F. Jarad, "Existence and uniqueness theorem for a class of delay differential equations with left and right Caputo fractional derivatives," Journal of Mathematical Physics, vol. 49, no. 8, Article ID 083507, 11 pages, 2008.

[17] T. Maraaba, F. Jarad, and D. Baleanu, "On the existence and the uniqueness theorem for fractional differential equations with bounded delay within Caputo derivatives," Science in China Series A, vol. 51, no. 10, pp. 1775-1786, 2008.

[18] R. Agarwal, B. de Andrade, and G. Siracusa, “On fractional integro-differential equations with statedependent delay," to appear in Computers \& Mathematics with Applications.

[19] Y. Hino, S. Murakami, and T. Naito, Functional-Differential Equations with Infinite Delay, vol. 1473 of Lecture Notes in Mathematics, Springer, Berlin, Germany, 1991.

[20] R. Agarwal, J. P. C. dos Santos, and C. Cuevas, "Analytic resolvent operator and existence results for fractional integro-differential equations," submitted.

[21] A. Granas and J. Dugundji, Fixed Point Theory, Springer Monographs in Mathematics, Springer, New York, NY, USA, 2003.

[22] R. H. Martin Jr., Nonlinear Operators and Differential Equations in Banach Spaces, Robert E. Krieger Publishing, Melbourne, Fla, USA, 1987. 